\title{
BMJ Open A clinical risk score to predict in- hospital mortality in critically ill patients with COVID-19: a retrospective cohort study
}

\author{
Salem Alkaabi, ${ }^{1,2}$ Asma Alnuaimi, ${ }^{2}$ Mariam Alharbi, ${ }^{3}$ Mohammed A Amari, ${ }^{2}$ \\ Rajiv Ganapathy, ${ }^{4}$ Imran Iqbal, ${ }^{3}$ Javaid Nauman (D) , ${ }^{5,6,7}$ Abderrahim Oulhaj (D) ${ }^{5,8}$
}

To cite: Alkaabi S, Alnuaimi A, Alharbi M, et al. A clinical risk score to predict in-hospital mortality in critically ill patients with COVID-19: a retrospective cohort study. BMJ Open 2021;11:e048770. doi:10.1136/ bmjopen-2021-048770

- Prepublication history and additional supplemental material for this paper are available online. To view these files, please visit the journal online. (http://dx.doi.org/10.1136/ bmjopen-2021-048770).

Received 06 January 2021 Accepted 13 August 2021

Check for updates

(c) Author(s) (or their employer(s)) 2021. Re-use permitted under CC BY-NC. No commercial re-use. See rights and permissions. Published by BMJ.

For numbered affiliations see end of article.

Correspondence to Dr Abderrahim Oulhaj; aoulhaj@uaeu.ac.ae

\section{ABSTRACT}

Objectives To identify factors influencing the mortality risk in critically ill patients with COVID-19, and to develop a risk prediction score to be used at admission to intensive care unit (ICU).

Design A multicentre cohort study.

Setting and participants 1542 patients with COVID-19 admitted to ICUs in public hospitals of Abu Dhabi, United Arab Emirates between 1 March 2020 and 22 July 2020. Main outcomes and measures The primary outcome was time from ICU admission until death. We used competing risk regression models and Least Absolute Shrinkage and Selection Operator to identify the factors, and to construct a risk score. Predictive ability of the score was assessed by the area under the receiver operating characteristic curve (AUC), and the Brier score using 500 bootstraps replications.

Results Among patients admitted to ICU, 196 (12.7\%) died, 1215 (78.8\%) were discharged and 131 (8.5\%) were right-censored. The cumulative mortality incidence was $14 \%$ (95\% Cl $12.17 \%$ to $15.82 \%)$. From 36 potential predictors, we identified seven factors associated with mortality, and included in the risk score: age (adjusted HR (AHR) 1.98; 95\% Cl 1.71 to 2.31), neutrophil percentage (AHR 1.71; 95\% Cl 1.27 to 2.31), lactate dehydrogenase (AHR 1.31; 95\% Cl 1.15 to 1.49), respiratory rate (AHR $1.31 ; 95 \% \mathrm{Cl} 1.15$ to 1.49), creatinine (AHR 1.19; $95 \%$ Cl 1.11 to 1.28), Glasgow Coma Scale (AHR 0.70; 95\% $\mathrm{Cl} 0.63$ to 0.78 ) and oxygen saturation $\left(\mathrm{SpO}_{2}\right)$ (AHR 0.82; $95 \% \mathrm{Cl} 0.74$ to 0.91$)$. The mean AUC was $88.1(95 \% \mathrm{Cl}$ 85.6 to 91.6$)$, and the Brier score was 8.11 (95\% Cl 6.74 to 9.60$)$. We developed a freely available web-based risk calculator (https://icumortalityrisk.shinyapps.io/ICUrisk/). Conclusion In critically ill patients with COVID-19, we identified factors associated with mortality, and developed a risk prediction tool that showed high predictive ability. This tool may have utility in clinical settings to guide decision-making, and may facilitate the identification of supportive therapies to improve outcomes.

\section{INTRODUCTION}

The COVID-19 pandemic caused by SARSCoV-2 has affected $>151$ million patients, and $>3.1$ million have died, as of 4 May 2021. ${ }^{1}$ A wide spectrum of clinical symptoms

\section{STRENGTHS AND LIMITATIONS OF THIS STUDY \\ $\Rightarrow$ Patients admitted to intensive care unit (ICU) with confirmed COVID-19 have relatively high prevalence of in-hospital mortality, however, limited data are available regarding the risk prediction scores in this population. \\ $\Rightarrow$ Our clinical risk score includes clinical features which are readily available at ICU admission, thus amplifying its clinical applicability. \\ $\Rightarrow$ A major limitation is the generalisability of risk pre- diction score to other settings, and external valida- tion should be the next step.}

of SARS-CoV-2 has been reported, encompassing asymptomatic infection, mild upper respiratory tract illness and severe viral pneumonia with respiratory failure leading to hospital admission and death. ${ }^{23}$

The preventive and treatment challenge of COVID-19 is very high because of the complexity of its transmission, substantial heterogeneity in the progression of disease and lack of proven treatment. ${ }^{4}{ }^{5}$ Several studies have attempted to address this by predicting clinical outcomes using statistical association analyses or prediction model development methods in order to guide the management and prognostication of patients with COVID-19. ${ }^{6-17}$ Based on patient characteristics at the time of hospital admission, Liang $e t a l^{7}$ proposed a risk score to predict critical illness defined as a composite of intensive care unit (ICU) admission, invasive ventilation or death. Similarly, a severity score ranging from 0 to 10 is proposed to predict inpatient mortality in patients with COVID19 , which consisted of six parameters assessed at the time of hospital admission. ${ }^{12}$

A modified Nutrition Risk in the Critically ill (mNUTRIC) score assessed at ICU admission has also shown higher mortality in patients with COVID-19 with high nutritional 
risk compared with those with low nutritional risk. ${ }^{14}$ Furthermore, a prognostic score using machine learning methods has been shown to predict death in ICU patients with COVID-19. ${ }^{15}$ Additionally, various demographics, clinical-level and hospital-level risk factors have been reported to be associated with death in patients admitted to ICU. ${ }^{8}$

A recent meta-analysis showed that more than onefourth of patients with COVID-19 were admitted to ICU globally, and the prevalence of mortality among these patients was very high $(31 \%) \cdot{ }^{18}$ However, limited data are available related to prognostic risk score of in-hospital mortality in critically ill patients with COVID-19 who were admitted to ICU. Therefore, the aim of the present study was to identify the risk factors and the set of clinical markers that increase the risk of death among ICUadmitted patients with COVID-19, and to develop a risk prediction score that may facilitate the identification of supportive therapies to improve outcomes. We also aim to develop an easy-to-use web-based risk calculator implementing the derived risk prediction score to allow clinicians enter the values of the selected variables required for the risk calculation of mortality in patients admitted to ICU with COVID-19. The online calculator will provide stratification of patients into high-risk and low-risk categories based on an estimated cut-off risk corresponding to optimal performance measures of sensitivity and specificity.

\section{METHODS}

\section{Study design and data sources}

This is a multicentre cohort study in which data of all laboratory confirmed patients with COVID-19 admitted to ICU in the Emirate of Abu Dhabi, United Arab Emirates (UAE) between 1 March 2020 and 22 July 2020 were retrieved from electronic medical records. The data were collected from four major hospitals as well as newly developed field hospitals operating with some ICU bed capacity. The estimated bed capacity for ICU and/or high-dependency unit (HDU) was around 550 across the Emirate. We included patients who were admitted to a regular ICU room or to a HDU or if they were consistently receiving any form of oxygen therapy during their hospital stay in a make-shift ICU.

\section{Outcomes}

The primary outcome of this study is the survival time defined as the duration of time, from the date of ICU admission, until the date of death. Patients still hospitalised at the date of data extraction were considered as right censored and those discharged alive from the hospital were considered as competing events to death due to COVID-19.

\section{Statistical analyses}

Baseline characteristics were summarised using descriptive statistics including mean and SD for continuous measures, and frequencies tables for categorical variables. We compared categorical variables using the $\chi^{2}$ or Fisher's exact test, and continuous variables using the unpaired t-test or its non-parametric equivalent (Wilcoxon rank sum test) in case the normality assumption is violated.

\section{Potential predictive variables}

We considered 36 patient's characteristics assessed at the time of ICU admission as potential predictors based on demographics, clinical signs and symptoms, medical history and laboratory findings. Demographic variables included age and sex. Clinical signs and symptoms included systolic blood pressure, diastolic blood pressure, respiratory rate, Glasgow Coma Scale ratings and minimum level of peripheral capillary oxygen saturation $\left(\mathrm{SpO}_{2}\right)$. Medical history included status of coexisting conditions: diabetes, hypertension, cardiovascular disease, respiratory diseases, chronic kidney disease, cancer and liver disease. Laboratory findings included white blood cells, monocytes count, monocytes percentage, neutrophils count, neutrophils percentage, lymphocytes count, lymphocytes percentage, red blood cell, platelets count, neutrophils-lymphocytes count ratio, neutrophils-to-lymphocytes percentage ratio, levels of $\mathrm{C}$ reactive protein, lactate dehydrogenase (LDH), ferritin, haemoglobin, haematocrit, sodium, potassium, chloride, bicarbonates, creatinine and red blood cell distribution width. Patients with available data on these characteristics were included in the final analysis.

\section{The statistical model}

We used the competing risk regression model to investigate the association between death due to COVID-19 and all potential risk factors. We have chosen to use this model, instead of the standard Cox proportional hazard model, because discharge alive or recovery is clearly a competing event to death due to COVID-19. ${ }^{19}{ }^{20}$ Ignoring this property will lead to biased estimates of the HRs and the survival curves. We estimated and plotted the survival curves using the cumulative incidence function taking into account competing risks. Cumulative incidence curves of different groups were compared using the Gray's test ${ }^{21}$ for subdistribution hazards, an equivalent of the log-rank test in the case of competing events. We used the Fine and Gray proportional hazards regression models $^{22}$ to investigate the association between potential risk factors and the primary outcome, and also to derive the risk prediction score. All statistical analysis and data management carried out in this paper were done using the R software V.3.6.3 and $p$ values $<0.05$ were considered as statistically significant.

\section{Variables selection method and derivation of the risk prediction} score

We used the Least Absolute Shrinkage and Selection Operator (LASSO) with Bayes Information criterion (BIC) for variables selection. ${ }^{23} 24$ This method uses a shrinking parameter to penalise non-significant coefficients of the 
Fine and Gray competing risk regression model. Larger shrinking parameters make the coefficients of nonsignificant risk factors to shrink towards zero, so that only the strongest predictors remain in the survival model. Unlike the standard selection methods, such as stepwise forward or backward, the LASSO procedure can deal with issues of multicollinearity. All the 36 potential predictors were scaled using the z-score transformation, and were entered in the selection process. The most predictive covariates were selected by choosing the shrinking parameter that minimises the BIC. Predictors selected by the LASSO procedure that were statistically significant were retained to construct the risk prediction score. We also investigated all statistical interactions between pairs of the retained predictors.

\section{Validation of the risk prediction score}

We derived the 28-day risk of in-hospital death using the estimates obtained from the Fine and Gray competing risk regression model. The predictive ability of this proposed risk prediction score was assessed using discrimination and calibration. Discrimination refers to how well the predictive model is capable of discriminating between individuals who died and those who were discharged alive, whereas calibration refers to the agreement between observed and predicted number of deaths. Discrimination was assessed via the time-dependent area under the receiver operating characteristic curve (AUC). Calibration was assessed via the time-dependent Brier score, and visually by plotting expected versus observed deaths. To reduce overfitting and optimism bias, we carried out internal validation of the risk prediction score by estimating the AUC and Brier score using 500 bootstraps replications. This method allows all of the original data to be used in the model development while providing insight into the extent to which the original model is overfitting or too optimistic.

\section{Patient and public involvement}

Patients or the public were not involved in the design, or conduct, or reporting, or dissemination plans of our research.

\section{Results}

A total of 1695 patients were eligible for the study entry among which 1542 had complete information on all the potential predictors and hence were included in the analysis. The characteristics of the 153 patients who were excluded from the analysis (due to missing values) were not different from those who were included in the current analysis (online supplemental eTable 1). Almost three-quarters of the study patients were Asians and nearly one-quarter were Arabs, which is consistent with the demographic composition of the entire population of Abu Dhabi. Of the 1542 patients with COVID-19 admitted to ICU, 196 (12.7 \%) died, 1215 (78.8\%) were discharged alive and 131 (8.5\%) were right-censored (ie, still hospitalised at the date of data extraction). Taking

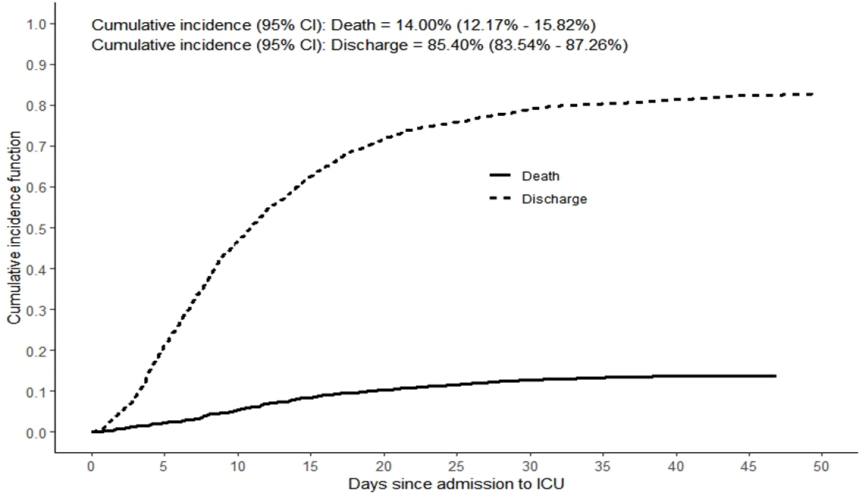

Figure 1 Mortality and recovery curves among critically ill patients with COVID-19. ICU, intensive care unit.

into account right-censored observations, the cumulative incidence of mortality was estimated at $14 \%(95 \%$ CI $12.17 \%$ to $15.82 \%$ ), and the cumulative incidence of discharge was estimated to be $85.40 \%$ (95\% CI 83.54 to 87.26) (figure 1).

The demographic and clinical characteristics of the patients are presented in table 1. Among 221 female patients, $28(12.7 \%)$ have died, and among 1321 male patients, 168 (12.7\%) have died. Compared with patients who were discharged alive, those who died were older and had higher prevalence of diabetes, hypertension, chronic kidney disease, cardiovascular disease and liver disease; lower diastolic blood pressure, higher respiratory rate, lower scores of Glasgow Coma Scale, lower levels of $\mathrm{SpO}_{2}$ and a higher percentage of patients requiring oxygen therapy.

The laboratory findings of the patients included in our study are presented in table 2. Compared with patients who were discharged alive, those who died had unfavourable laboratory profile on almost all variables including levels of $\mathrm{C}$ reactive protein, creatinine, $\mathrm{LDH}$, red blood cell distribution width, white blood cell count, potassium, ferritin, values of red blood cells, lymphocytes, monocytes, platelets count, haemoglobin, haematocrit and serum bicarbonates.

The results of the univariate competing risk model for each of the 36 potential predictors measured at ICU admission are presented in the online supplemental eTable 2. Of these 36 variables, 7 statistically significant predictors of mortality were retained by the LASSO selection procedure in the multivariable competing risk regression model (online supplemental eFigure 1). The HRs, $\mathrm{p}$ values and $95 \%$ CIs of these significant variables are presented in table 3 . The significant predictors increasing the risk of death included older age (HR 1.98 (95\% CI 1.71 to 2.31$) ; \mathrm{p}<0.001)$, higher neutrophil percentage (HR 1.71 (95\% CI 1.27 to 2.31); $<<0.001$ ), higher $\mathrm{LDH}$ levels (HR 1.31 (95\% CI 1.15 to 1.49 ); $\mathrm{p}<0.001$ ), higher respiratory rate (HR 1.31 (95\% CI 1.15 to 1.49$)$; $\mathrm{p}<0.001)$ and high levels of creatinine (HR 1.19 (95\% CI 1.11 to $1.28) ; \mathrm{p}<0.001)$. The significant predictors lowering the risk of death included higher Glasgow Coma Scale (HR 
Table 1 Demographics and clinical characteristics among critically ill patients with COVID-19

\begin{tabular}{|c|c|c|c|c|}
\hline Characteristics & Total & Died & Alive & P value* \\
\hline Female sex, n (\%) & $221(14.3)$ & $28(14.3)$ & $193(14.3)$ & \\
\hline $\begin{array}{l}\text { Systolic blood pressure, mean (SD), mm } \\
\mathrm{Hg}\end{array}$ & $126.2(17.4)$ & $125.5(21.1)$ & $126.3(16.8)$ & 0.286 \\
\hline Respiratory rate, mean (SD), breaths/min & $23.3(6.7)$ & $27.1(7.4)$ & $22.7(6.4)$ & $<0.001$ \\
\hline \multicolumn{5}{|l|}{ Oxygen saturation $\left(\mathrm{SpO}_{2}\right), \mathrm{n}(\%)$} \\
\hline$<90$ & $487(31.6)$ & $138(70.4)$ & $349(25.9)$ & \\
\hline $90-94$ & $569(36.9)$ & $39(19.9)$ & $530(39.4)$ & \\
\hline $\begin{array}{l}\text { Hypoxic respiratory failure requiring } \\
\text { non-invasive mechanical ventilation }\end{array}$ & $76(4.9)$ & $11(5.6)$ & $65(4.8)$ & \\
\hline $\begin{array}{l}\text { Hypoxic respiratory failure requiring } \\
\text { invasive mechanical ventilation }\end{array}$ & $519(33.7)$ & $167(85.2)$ & $352(26.2)$ & $<0.001$ \\
\hline \multicolumn{5}{|l|}{ Co-existing conditions, n (\%) } \\
\hline 0 & $498(32.3)$ & 39 (19.9) & $459(34.1)$ & \\
\hline 1 & $403(26.1)$ & $45(23.0)$ & $358(26.6)$ & \\
\hline$\geq 2$ & $641(41.6)$ & $112(57.1)$ & $529(39.3)$ & $<0.001$ \\
\hline \multicolumn{5}{|l|}{ Respiratory disease, n (\%) } \\
\hline No & $1361(88.3)$ & $166(84.7)$ & $1195(88.8)$ & \\
\hline Yes & $181(11.7)$ & $30(15.3)$ & $151(11.2)$ & 0.123 \\
\hline \multicolumn{5}{|l|}{ Cardiovascular disease, n (\%) } \\
\hline No & $1053(68.3)$ & $100(51.0)$ & $953(70.8)$ & \\
\hline Yes & $489(31.7)$ & $96(49.0)$ & $393(29.2)$ & $<0.001$ \\
\hline \multicolumn{5}{|l|}{ Chronic kidney disease, n (\%) } \\
\hline No & $1397(90.6)$ & $159(81.1)$ & $1238(92.0)$ & \\
\hline Yes & $145(9.4)$ & $37(18.9)$ & $108(8.0)$ & $<0.001$ \\
\hline \multicolumn{5}{|l|}{ Cancer, n (\%) } \\
\hline No & 1479 (95.9) & $183(93.4)$ & $1296(96.3)$ & \\
\hline Yes & $63(4.1)$ & $13(6.6)$ & $50(3.7)$ & 0.083 \\
\hline \multicolumn{5}{|l|}{ Liver disease, n (\%) } \\
\hline No & $1437(93.2)$ & $174(88.8)$ & $1263(93.8)$ & \\
\hline Yes & $105(6.8)$ & $22(11.2)$ & $83(6.2)$ & 0.013 \\
\hline Glasgow Coma Scale, mean (SD) & $13.83( \pm 3.42)$ & $11.94( \pm 5.07)$ & $14.28( \pm 2.71)$ & $<0.001$ \\
\hline Mild, n (\%) & $1391(90.2)$ & $121(61.7)$ & $1270(94.4)$ & \\
\hline
\end{tabular}


Table 1 Continued

\begin{tabular}{lcccc}
\hline Characteristics & Total & Died & Alive & P value* \\
\hline Moderate, $\mathrm{n}(\%)$ & $21(1.4)$ & $7(3.6)$ & $14(1.0)$ & $62(4.6)$
\end{tabular}

Glasgow Coma Scale: mild (14-15), moderate (9-13) or severe (3-8).

${ }^{*}$ Continuous variables were compared using the t-test or Wilcoxon rank sum test, while categorical variables were compared using the $\chi^{2}$ or Fisher's exact test.

†The percentages do not sum up to $100 \%$ because there are patients not requiring any form of oxygen therapy.

$0.70(95 \%$ CI 0.63 to 0.78$) ; \mathrm{p}<0.001)$ and higher $\mathrm{SpO}_{2}$ levels (HR 0.82 (95\% CI 0.74 to 0.91); $<<0.001$ ). We found no statistically significant interaction terms between pairs of the retained predictors.

The cumulative incidence function of these seven predictors retained by LASSO in the multivariable model is shown in the online supplemental eFigure 2. For graphical presentation, we created a binary variable based on the median split in case of continuous risk factors.

\section{Validation of the risk prediction score}

The results of the internal validation using 500 bootstrap samples are shown in figure 2. The predictive ability of the derived risk prediction score was quite promising. Indeed, regarding discrimination, the estimated AUC was
88.1 (95\% CI 85.6 to 90.6), and the Brier score, measuring calibration, was estimated to 8.11 (95\% CI 6.74 to 9.60). Figure 2 also shows the calibration plot for the risk prediction score, in which the predicted frequencies of deaths were plotted against the observed ones.

From figure 2, it is evident that the predicted frequencies of death were very close to the observed ones suggesting a very good calibration. The risk prediction score provided a sensitivity of $81 \%$ and a specificity of $79 \%$ using a cut-off risk of $11.5 \%$.

We also developed an easy-to-use web-based risk calculator implementing the derived risk prediction score to allow clinicians enter the values of the selected variables required for the risk calculation of mortality in patients

Table 2 Laboratory findings among critically ill patients with COVID-19

\begin{tabular}{|c|c|c|c|c|}
\hline Variable & Total & Died & Alive & \\
\hline Number (\%) & $1542(100)$ & $196(13)$ & $1346(87)$ & $P$ value \\
\hline White blood cells, mean (SD), $\times 10^{9} / \mathrm{L}$ & 7.89 (3.92) & $10.40(6.01)$ & $7.52(3.36)$ & $<0.001$ \\
\hline Lymphocyte per cent, mean (SD), \% & $18.76(10.74)$ & $10.86(6.93)$ & $19.92(10.72)$ & $<0.001$ \\
\hline Neutrophil count, mean (SD), $\times 10^{9} / \mathrm{L}$ & $6.00(3.77)$ & $8.85(5.65)$ & $5.58(3.21)$ & $<0.001$ \\
\hline Neutrophil-to-lymphocyte count ratio & $6.81(9.39)$ & $13.66(18.16)$ & $5.82(6.73)$ & $<0.001$ \\
\hline Neutrophil-to-lymphocyte per cent ratio & $6.82(9.46)$ & $13.69(18.33)$ & $5.82(6.77)$ & $<0.001$ \\
\hline Monocytes count, mean (SD), $\times 10^{9} / \mathrm{L}$ & $0.51(0.36)$ & $0.50(0.72)$ & $0.51(0.28)$ & 0.769 \\
\hline Monocytes per cent, mean (SD), \% & $6.93(3.67)$ & $4.94(4.99)$ & $7.22(3.34)$ & $<0.001$ \\
\hline Platelet count, mean (SD), $\times 10^{9} / \mathrm{L}$ & 263.57 (107.94) & $241.88(104.12)$ & $266.72(108.17)$ & 0.002 \\
\hline Haemoglobin level, mean (SD), g/L & $131.90(18.24)$ & $126.18(19.59)$ & $132.73(17.89)$ & $<0.001$ \\
\hline Haematocrit, mean (SD), L/L & $0.39(0.05)$ & $0.38(0.06)$ & $0.40(0.05)$ & $<0.001$ \\
\hline Creatinine level, mean (SD), $\mu \mathrm{mol} / \mathrm{L}$ & $97.76(111.80)$ & $149.97(188.91)$ & $90.15(93.23)$ & $<0.001$ \\
\hline$C$ reactive protein level, mean $(S D), \mathrm{mg} / \mathrm{L}$ & $102.13(94.00)$ & $173.05(112.06)$ & $91.80(86.39)$ & $<0.001$ \\
\hline Lactate dehydrogenase, mean (SD), IU/L & $409.32(223.16)$ & $605.80(291.22)$ & $380.71(195.74)$ & $<0.001$ \\
\hline Serum chloride, mean (SD), mmol/L & $99.25(4.50)$ & $99.05(5.97)$ & $99.28(4.25)$ & 0.600 \\
\hline Serum bicarbonate, mean (SD), $\mathrm{mmol} / \mathrm{L}$ & $22.80(3.28)$ & $21.02(4.23)$ & $23.06(3.04)$ & $<0.001$ \\
\hline Potassium, mean (SD), mmol/L & $4.05(0.55)$ & $4.21(0.76)$ & $4.03(0.50)$ & 0.002 \\
\hline Sodium, mean (SD), mmol/L & $136.98(4.36)$ & $136.97(5.80)$ & $136.98(4.11)$ & 0.984 \\
\hline
\end{tabular}


Table 3 Multivariable adjusted competing risk regression model for mortality

\begin{tabular}{|c|c|c|}
\hline Variables & HR (95\% Cl) & $P$ value \\
\hline Age, years & 1.98 (1.71 to 2.31$)$ & $<0.001$ \\
\hline $\begin{array}{l}\text { Neutrophils } \times 10^{9} / \mathrm{L} \text {, } \\
\text { percentage }\end{array}$ & $1.71(1.27$ to 2.31$)$ & $<0.001$ \\
\hline $\begin{array}{l}\text { Lactate dehydrogenase, } \\
\text { IU/L }\end{array}$ & 1.31 (1.15 to 1.49$)$ & $<0.001$ \\
\hline $\begin{array}{l}\text { Respiratory rate, } \\
\text { breaths/min }\end{array}$ & 1.31 (1.15 to 1.49$)$ & $<0.001$ \\
\hline Glasgow Coma Scale & $0.70(0.63$ to 0.78$)$ & $<0.001$ \\
\hline Oxygen saturation $\left(\mathrm{SpO}_{2}\right)$ & 0.82 (0.74 to 0.91$)$ & $<0.001$ \\
\hline Creatinine, $\mu \mathrm{mol} / \mathrm{L}$ & $1.19(1.11$ to 1.28$)$ & $<0.001$ \\
\hline \multicolumn{3}{|c|}{$\begin{array}{l}\text { The predictors were scaled using z-score transformation, and } \\
\mathrm{HRs} \text { should be interpreted as } 1 \mathrm{SD} \text { change in the values of the } \\
\text { parameters. } \\
\mathrm{SpO}_{2} \text {, peripheral capillary oxygen saturation. }\end{array}$} \\
\hline
\end{tabular}

admitted to ICU with COVID-19. The online calculator also provides stratification of patients into high-risk and low-risk categories based on an estimated cut-off risk corresponding to optimal performance measures of sensitivity and specificity. The online risk calculator is freely available at (https://icumortalityrisk.shinyapps.io/ ICUrisk/).

\section{Discussion}

We developed and internally validated a clinical risk prediction score and a web-based risk calculator to predict the risk of in-hospital death in adult patients with confirmed COVID-19 admitted to ICUs. The risk prediction score shows high accuracy in terms of discrimination $(\mathrm{AUC}=88.1)$ and calibration (Bier score $=8.11$ ) with an almost perfect similarity between predicted and expected deaths. We identified seven readily available clinical features at ICU admission to be used for risk prediction of in-hospital mortality namely age, minimum oxygen saturation, respiratory rate, Glasgow Coma Scale ratings, neutrophil percentage, LDH and creatinine levels. Our work shows that input of these variables in an easy-to use web-based risk calculator has the potential to accurately classify ICU admitted patients as likely to be discharged alive or die.

A major strength of this study is the relatively large number of laboratory confirmed patients with COVID-19 admitted to ICU, and the inclusion of information on a broad range of demographic, clinical and laboratory characteristics. Furthermore, the risk prediction score includes clinical features that are readily available at ICU admission that increases its clinical applicability. An obvious limitation of this study is the generalisability of risk prediction score in other settings, and we acknowledge that external validation of our risk prediction score in other populations is the next step in model development. Furthermore, the participants included in this study were younger compared with other studies using the data at the time of hospital admission, ${ }^{812-17}$ which may in turn limit the generalisability in older patients.

Previous studies have reported risk prediction scores of mortality based on the clinical features at the time of hospital or ICU admission, including patients with mild, moderate or severe forms of disease. ${ }^{6791012-17}$ For instance, using data of 4711 confirmed patients with COVID-19, a severity score to predict in-hospital mortality was developed and validated, and consisted of six variables (age, oxygen saturation, mean arterial pressure, blood urea nitrogen, $\mathrm{C}$ reactive protein and the international normalised ratio) assessed at the time of hospital admission. ${ }^{12}$ Moreover, 10 variables (chest radiographic abnormality, age, haemoptysis, dyspnoea, unconsciousness,

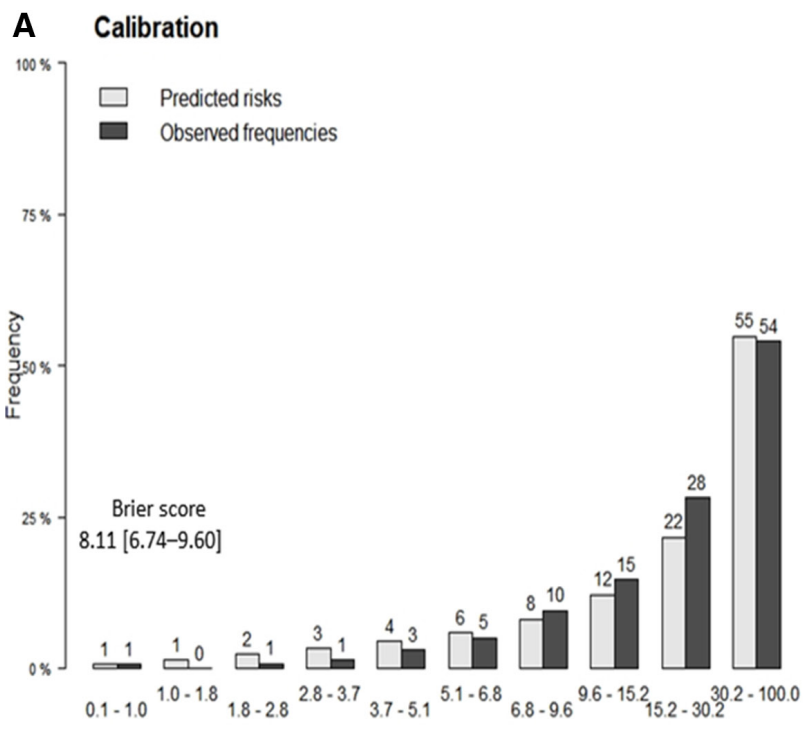

B Discrimination

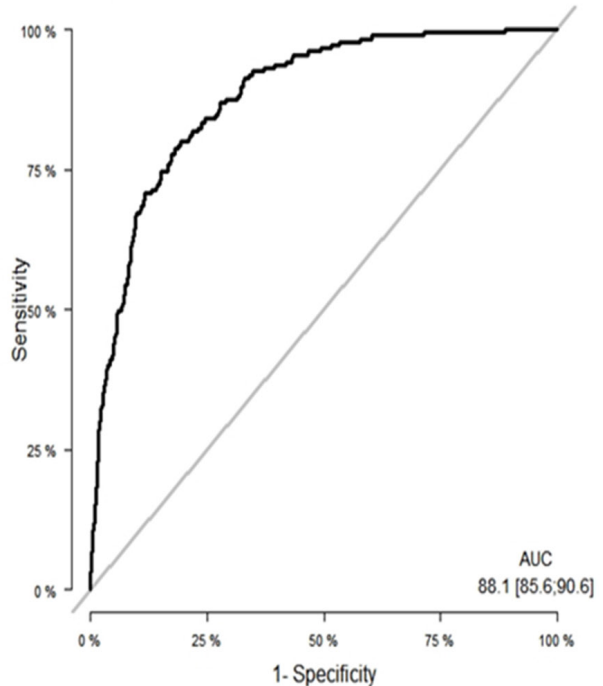

Figure 2 (A) Calibration and (B) area under the receiver operating characteristic curve (AUC) of predicting death among patients with COVID-19 admitted to ICU. 
number of comorbidities, cancer history, neutrophil-tolymphocyte ratio, $\mathrm{LDH}$ and direct bilirubin) were found to be independent predictive factors, and were included in the risk score to predict the occurrence of critical illness in hospitalised patients with COVID-19. ${ }^{7}$ The International Severe Respiratory and emerging Infections Consortium developed and validated a mortality score consisting of eight variables (age, sex, number of comorbidities, respiratory rate, peripheral oxygen saturation, level of consciousness, urea level and $\mathrm{C}$ reactive protein) that were available at the initial hospital assessment. ${ }^{9}$ In line with this, methods using machine learning have identified eight important risk factors to predict mortality in ICU admitted patients with COVID-19. ${ }^{15}$ Interestingly, nutritional status of the critically ill patients with COVID-19 ascertained by mNUTRIC score at the time of ICU admission predicted twice the probability of death in patients with high nutritional risk than low-risk patients. ${ }^{14}$ The difference in the number and types of independent clinical features associated with mortality between our study and others may be explained by the differences in the baseline characteristics of the population or the choice of the statistical analyses. Indeed, we have chosen to use the competing risk regression model instead of the standard Cox proportional hazard model or the logistic regression model because recovery is clearly a competing event to in-hospital death due to COVID-19. ${ }^{19}{ }^{20}$ Ignoring this property will definitely lead to biased effect estimates. Another plausible reason for this difference in the results is the younger age of the participants in our study compared with other studies, ${ }^{12-17}$ which could likely influence the clinical features to be included in the risk prediction score.

Other statistical association analyses have been published to investigate the factors affecting mortality due to COVID-19 in patients admitted to ICU. ${ }^{811}$ For instance, a multicentre cohort study of 2215 adults with laboratory-confirmed COVID-19 admitted to ICU in the USA identified 9 risk factors independently associated with the 28 days mortality. These risk factors included age, sex, body mass index, coronary artery disease, active cancer, presence of hypoxaemia, liver dysfunction, kidney dysfunction and the number of hospital ICU beds. ${ }^{8}$ In our risk score, none of the comorbid conditions achieved statistical significance for in-hospital mortality, however, other significant laboratory findings such as increased $\mathrm{LDH}$ and increased creatinine levels may represent underlying diseases such as liver disease, lung disease or kidney dysfunction.

Interestingly, a non-COVID-19 prediction score named Waterlow score has shown to predict 30-day mortality and length of hospital stay in acutely admitted elderly patients. ${ }^{25}$ The Waterlow score is a multidimensional pressure ulcer risk assessment tool and includes age, nutritional status, weight, mobility, gender, smoking status, comorbidities, use of medication and continence. ${ }^{25}$ One of the significant predictors of mortality included in our risk score is Glasgow Coma Scale, which is an objective and reliable way of recording the initial and subsequent level of consciousness, and could be used as a proxy to continence. Although the association between Waterlow score and mortality is demonstrated in patients aged 65 years and above, especially for respiratory, cardiac and stroke conditions, its application in patients with confirmed COVID-19 warrants further investigations.

The recent COVID-19 epidemiological update from WHO, as of 4 May 2021, reported over 5.7 million new weekly cases worldwide which is at the highest level since the beginning of the pandemic. ${ }^{1}$ The WHO European and American regions accounted for $20 \%$ and $23 \%$ of new weekly cases, respectively. The largest increase accounting for $47 \%$ of new weekly cases was noted in South East Asia region particularly in India which accounted for over $90 \%$ of both cases and deaths in the region. The Eastern Mediterranean region that includes UAE accounted for $6 \%$ of new weekly cases. ${ }^{1}$ Earlier studies have reported rate of admission to ICU among confirmed SARS-CoV-2 cases ranging between $2 \%$ and $81 \%,{ }^{18} 2627$ and high mortality prevalence among ICU patients ranging between 5\% and 83\%. ${ }^{318} 28$ A metaanalysis of 25 studies with 24677 patients demonstrated a rate of $26 \%$ for ICU admission, and $31 \%$ mortality prevalence among patients admitted to ICU with a severe form of COVID-19. ${ }^{18}$ The relative high number of deaths in the ICU presents an enormous challenge to the prognostication and management of patients with COVID-19. We believe that the risk tool provided in this study may have utility in clinical settings to guide decision-making, and may facilitate the early identification of patients at high risk of death, and may be used as a guidance in busy ICU units to stratify patients according to their risk in order to deliver the best available supportive care. The parameters selected are easily available at the time of ICU admission.

\section{CONCLUSION}

We developed and internally validated a risk tool for predicting in-hospital death among patients with COVID-19 admitted to ICU, which shows high predictive accuracy. This tool can assist in early identification of patients during ICU admission who are at high risks of death, and consequently can facilitate optimal delivery of supportive care for these patients.

\section{Author affiliations}

'Zayed Military Hospital, Abu Dhabi, UAE

${ }^{2}$ Sheikh Khalifa Medical City, Abu Dhabi, UAE

${ }^{3}$ SEHA, Abu Dhabi Health Services Co, Abu Dhabi, UAE

${ }^{4}$ Cerner Middle East \& Africa, Dubai, UAE

${ }^{5}$ Institute of Public Health, College of Medicine and Health Sceinces, United Arab Emirates University, Al-Ain, UAE

${ }^{6}$ Department of Circulation and Medical Imaging, Faculty of Medicine and Health Sciences, Norwegian University of Science and Technology, Trondheim, Norway ${ }^{7}$ Healthy Living for Pandemic Event Protection (HL - PIVOT) Network, Chicago, IL, USA

${ }^{8}$ Zayed Center for Health Sciences, United Arab Emirates University, AI-Ain, UAE 
Acknowledgements The authors would like to thank Dr Fayez Al Shamsi for interesting discussions and suggestions that improved the paper.

Contributors SA, AA, MAH, MAA, RG, II, JN and AO contributed to the development of the research question and study design. $\mathrm{AO}$ led the development of advanced statistical aspects. SA, AA, MAH, MAA, RG, II and AO were involved in data specification, curation and collection. A0 did data management and statistical analyses, which were checked by SA, AA, MAH and JN. SA, AA, MAH, MAA, RG, II, JN and $A 0$ contributed to the interpretation of the results. $S A, A A, J N$ and $A 0$ wrote the first draft of the paper. SA, AA, MAH, MAA, RG, II, JN and AO contributed to the critical revision of the manuscript for important intellectual content and approved the final version of the manuscript. A0 developed the software for the web calculator. The corresponding author attests that all listed authors meet authorship criteria and that no others meeting the criteria have been omitted. $A 0$ is the guarantor. The lead author affirms that the manuscript is an honest, accurate and transparent account of the study being reported; that no important aspects of the study have been omitted and that any discrepancies from the study as planned (and, if relevant, registered) have been explained.

Funding The authors have not declared a specific grant for this research from any funding agency in the public, commercial or not-for-profit sectors.

Competing interests None declared.

Patient consent for publication Not required.

Ethics approval The study was approved by the Department of Health of Abu Dhabi COVID-19 IRB ethical committee (Ref\#DOH/CVDC/2020/1116).

Provenance and peer review Not commissioned; externally peer reviewed.

Data availability statement Data are available on reasonable request. Data may be obtained from a third party and are not publicly available. To guarantee the confidentiality of personal and health information, only the authors have had access to the data during the study in accordance with the relevant licence agreements. Access to the data is according to the information and rules and regulations of Abu Dhabi Health Services - SEHA and Cerner.

Supplemental material This content has been supplied by the author(s). It has not been vetted by BMJ Publishing Group Limited (BMJ) and may not have been peer-reviewed. Any opinions or recommendations discussed are solely those of the author(s) and are not endorsed by BMJ. BMJ disclaims all liability and responsibility arising from any reliance placed on the content. Where the content includes any translated material, BMJ does not warrant the accuracy and reliability of the translations (including but not limited to local regulations, clinical guidelines, terminology, drug names and drug dosages), and is not responsible for any error and/or omissions arising from translation and adaptation or otherwise.

Open access This is an open access article distributed in accordance with the Creative Commons Attribution Non Commercial (CC BY-NC 4.0) license, which permits others to distribute, remix, adapt, build upon this work non-commercially, and license their derivative works on different terms, provided the original work is properly cited, appropriate credit is given, any changes made indicated, and the use is non-commercial. See: http://creativecommons.org/licenses/by-nc/4.0/.

\section{ORCID iDs}

Javaid Nauman http://orcid.org/0000-0003-2813-9795

Abderrahim Oulhaj http://orcid.org/0000-0002-5330-904X

\section{REFERENCES}

1 World Health Organization. Coronavirus disease (COVID-19) Weekly epidemiological update and Weekly operational update, 2021. Available: https://www.who.int/emergencies/diseases/novelcoronavirus-2019/situation-reports [Accessed 10 May 2021].

2 Guan W-J, Ni Z-Y, Hu Y, et al. Clinical characteristics of coronavirus disease 2019 in China. N Engl J Med 2020;382:1708-20.

3 Richardson S, Hirsch JS, Narasimhan M, et al. Presenting characteristics, comorbidities, and outcomes among 5700 patients hospitalized with COVID-19 in the new York City area. JAMA 2020;323:2052-9.

4 Yang $\mathrm{X}, \mathrm{Yu}$ Y, Xu J, et al. Clinical course and outcomes of critically ill patients with SARS-CoV-2 pneumonia in Wuhan, China: a singlecentered, retrospective, observational study. Lancet Respir Med 2020;8:475-81.
5 Chen N, Zhou M, Dong X, et al. Epidemiological and clinical characteristics of 99 cases of 2019 novel coronavirus pneumonia in Wuhan, China: a descriptive study. Lancet 2020;395:507-13.

6 Wynants L, Van Calster B, Collins GS, et al. Prediction models for diagnosis and prognosis of covid-19: systematic review and critical appraisal. BMJ 2020;369:m1328.

7 Liang $\mathrm{W}$, Liang $\mathrm{H}$, Ou L, et al. Development and validation of a clinical risk score to predict the occurrence of critical illness in hospitalized patients with COVID-19. JAMA Intern Med 2020;180:1081-9.

8 Gupta S, Hayek SS, Wang W, et al. Factors associated with death in critically ill patients with coronavirus disease 2019 in the US. JAMA Intern Med 2020;180:1436.

9 Knight SR, Ho A, Pius R, et al. Risk stratification of patients admitted to hospital with covid-19 using the ISARIC who clinical characterisation protocol: development and validation of the $4 \mathrm{C}$ mortality score. BMJ 2020;370:m3339.

10 Zhang S, Guo M, Duan L, et al. Development and validation of a risk factor-based system to predict short-term survival in adult hospitalized patients with COVID-19: a multicenter, retrospective, cohort study. Crit Care 2020;24:438.

11 Noor FM, Islam MM. Prevalence and associated risk factors of mortality among COVID-19 patients: a meta-analysis. J Community Health 2020;45:1270-82.

12 Altschul DJ, Unda SR, Benton J, et al. A novel severity score to predict inpatient mortality in COVID-19 patients. Sci Rep 2020;10:16726

13 Zhao Z, Chen A, Hou W, et al. Prediction model and risk scores of ICU admission and mortality in COVID-19. PLoS One 2020;15:e0236618.

14 Zhang P, He Z, Yu G, et al. The modified NUTRIC score can be used for nutritional risk assessment as well as prognosis prediction in critically ill COVID-19 patients. Clin Nutr 2021;40:534-41.

15 Pan $\mathrm{P}, \mathrm{Li}$ Y, Xiao Y, et al. Prognostic assessment of COVID-19 in the intensive care unit by machine learning methods: model development and validation. J Med Internet Res 2020;22:e23128.

16 Fumagalli C, Rozzini R, Vannini M, et al. Clinical risk score to predict in-hospital mortality in COVID-19 patients: a retrospective cohort study. BMJ Open 2020;10:e040729.

17 Galloway JB, Norton S, Barker RD, et al. A clinical risk score to identify patients with COVID-19 at high risk of critical care admission or death: an observational cohort study. J Infect 2020;81:282-8.

18 Abate SM, Ahmed Ali S, Mantfardo B, et al. Rate of intensive care unit admission and outcomes among patients with coronavirus: a systematic review and meta-analysis. PLoS One 2020;15:e0235653.

19 McCaw ZR, Tian L, Vassy JL, et al. How to quantify and interpret treatment effects in comparative clinical studies of COVID-19. Ann Intern Med 2020;173:632-7.

20 Oulhaj Abderrahim AAL, Juergen P, Abubaker S, et al. The competing risk between in-hospital mortality and recovery: a pitfall in COVID-19 survival analysis research 2020.

21 GrayRJ. A class of K-Sample tests for comparing the cumulative incidence of a competing risk. The Annals of Statistics 1988:1141-54.

22 Fine JP, Gray RJ. A proportional hazards model for the Subdistribution of a competing risk. J Am Stat Assoc 1999;94:496-509.

23 Gui J, Li H. Penalized Cox regression analysis in the highdimensional and low-sample size settings, with applications to microarray gene expression data. Bioinformatics 2005;21:3001-8

24 Park MY. Hastie T. L1-regularization path algorithm for generalized linear models. Journal of the Royal Statistical Society: Series B 2007;69:659-77.

25 Wang JW, Smith P, Sarker S-J, et al. Can Waterlow score predict 30day mortality and length of stay in acutely admitted medical patients (aged $\geq 65$ years)? Evidence from a single centre prospective cohort study. BMJ Open 2019;9:e032347.

$26 \mathrm{Xu} \mathrm{X-W,} \mathrm{Wu} \mathrm{X-X,} \mathrm{Jiang} \mathrm{X-G,} \mathrm{et} \mathrm{al.} \mathrm{Clinical} \mathrm{findings} \mathrm{in} \mathrm{a} \mathrm{group}$ of patients infected with the 2019 novel coronavirus (SARSCov-2) outside of Wuhan, China: retrospective case series. BMJ 2020;368:m606.

27 Arentz M, Yim E, Klaff L, et al. Characteristics and outcomes of 21 critically ill patients with COVID-19 in Washington state. JAMA 2020;323:1612-4.

28 Wu C, Chen X, Cai Y, et al. Risk factors associated with acute respiratory distress syndrome and death in patients with coronavirus disease 2019 pneumonia in Wuhan, China. JAMA Intern Med 2020;180:934-43. 